NUCLEAR ENERGY UNIVERSITY PROGRAMS

\title{
Advanced Models of LWR Pressure Vessel Embrittlement for Low Flux-High Fluence Conditions
}

\author{
PI: Odette, G. Robert - University of \\ California, Santa Barbara
}

Project Number: 09-834

Initiative: LWRS

\author{
Collaborators: \\ Nanstad, Randy K. - Oak Ridge National \\ Laboratory \\ Wirth, Brian D. - University of California, \\ Berkeley \\ Yamamoto, Takuya - University of \\ California, Santa Barbara
}

\begin{abstract}
$\underline{\text { Abstract }}$
Neutron embrittlement of reactor pressure vessels (RPVs) is an unresolved issue for light water reactor life extension, especially since transition temperature shifts (TTS) must be predicted for high 80 -year fluence levels up to approximately $1,020 \mathrm{n} / \mathrm{cm}^{2}$, far beyond the current surveillance database. Unfortunately, TTS may accelerate at high fluence, and may be further amplified by the formation of late blooming phases that result in severe embrittlement even in low-copper $(\mathrm{Cu})$ steels. Embrittlement by this mechanism is a potentially significant degradation phenomenon that is not predicted by current regulatory models. This project will focus on accurately predicting transition temperature shifts at high fluence using advanced physically based, empirically validated and calibrated models. A major challenge is to develop models that can adjust test reactor data to account for flux effects. Since transition temperature shifts depend on synergistic combinations of many variables, flux-effects cannot be treated in isolation.

The best current models systematically and significantly under-predict transition temperature at high fluence, although predominantly for irradiations at much higher flux than actual RPV service. This project will integrate surveillance, test reactor and mechanism data with advanced models to address a number of outstanding RPV embrittlement issues. The effort will include developing new databases and preliminary models of flux effects for irradiation conditions ranging from very low (e.g., boiling water reactor) to high (e.g., accelerated test reactor). The team will also develop a database and physical models to help predict the conditions for the formation of $\mathrm{Mn}-\mathrm{Ni}-\mathrm{Si}$ late blooming phases and to guide future efforts to fully resolve this issue. Researchers will carry out other tasks on a best-effort basis, including prediction of transition temperature shift attenuation through the vessel wall, remediation of embrittlement by annealing, and fracture toughness master curve issues.
\end{abstract}

